Gynäk. Rdsch. 1985;25(suppl. 3):I-II

\title{
Contents, Vol. 25, Supplement 3, 1985
}

\section{Index}

2. Hauptthema $\cdot 2$ e sujet principall

\section{Urininkontinenz}

L'incontinence urinaire

Präsident · President: E. Hochuli, Münsterlingen

Ulmsten U.: Physiologie der Kontinenz und Pathophysiologie der Stressinkontinenz

Eberhard, J.: Urodynamische Definitionen 15

DeGrandi, P.: Procédés diagnostiques actuels de Гincontinence urinaire

Hochuli, E.: Operatives Therapiekonzept 38

Petri, E.: Die konservative Behandlung der Funktionsstörungen des unteren Harntraktes 47

Mitteilungen zum 2. Hauptthema Communications se rapportant au 2e sujet principal Eberhard, J.; Hotz, V.; Meyer, A.: Auswertung der Urethradruckprofile nach den neuen Kriterien der Schweizerischen Arbeitsgruppe für Urodynamik 53 Keller, H.J.; Eberhard, J.: Beurteilung der operationsbedingten Veränderungen der Urethraverschlussfunktion mit zwei verschiedenen Auswertungsmethoden . . 56 Wenger, M.; Eberhard, J.: Topographische Veränderungen nach UrethrovesikopexieOperationen 60

Casper, F.; Petri, E.: Sind kleine urodynamische Messplätze konkurrenzfähig? .... 63 1 Die Referate zum 1. Hauptthema «Strafrechtliche und zivilrechtliche Haftung des Arztes in der Geburtshilfe» sind erschienen in der Schweizerischen Arztezeitung 66: 1649- 1664(1985).

Les exposes concernant le ler sujet principal: «La responsabilité pénale et civile du medecin en obstétrique» ont paru dans le Bulletin des Medecins suisses 66: 1649-1664 (1985).

Index II

Freie Mitteilungen · Communications libres

Perinatologie/Geburtshilfe

Périnatologie/Obstétrique

Vetter, K.; Balas, G.; Fallenstein, F.; Huch, R.; Huch, A.: CO-Haemoglobinbestimmung in einer Population schwangerer Frauen 66

Schwöbel, E.; Ehrat, R.: Eine Zwillingsschwangerschaft mit ausgeprägter Grössendiskrepanz in der 17. Schwangerschaftswoche 68

Gigon, U.; Chu-Chen, A.; Pescia, G.; Nguyen-The, H.; Aufdermauer, P.: Zum Pro blem von dizygoten Zwillingsschwangerschaften mit pränataler Diagnose eines gesunden und eines kranken Feten 71

Baer, S.; Bung, P.; Djahanschahi, D.; Vetter, K.; Huch, R.; Huch, A.: Multizentrische Studie über die Geburtseinleitung mit intrazervikal appliziertem PGE2-Triacetin-Gel 75 
Germond, M.; Von Moos, G.; Marcos, J.P.; Mayer, Ph.; Calame, A.; Bossart, H.:

Mode d'accouchement des grossesses gémellaires pendant la période 1974-1983

à la Maternité du CHUV, Lausanne 77

Gynäkologie Gynécologie

Cavin, C.; Riedo, R.; Samartzis, S.; Hauser, G.A.: Einfluss des Alters und der Therapiedaueraufden Erfolgbei 1296 Sterilitätsfallen 79

Meandzija, M.P.; Locher, G.W.; Dreher, E.; Berger, M.: COi-Laser-Verdampfung bei intraepithelialer vaginaler Neoplasie 82

Maroni, E.; Angst, F.: Das Mammakarzinom in der Schwangerschaft $\quad 84$

Bronz, L.; Genton, C.Y.; Wyss, M.; Otto, R.; Schreiner, W.E.: Ein Fall von Adeno-

karzinom der Vagina bei Diäthylstilbestrol-Exposition in utero $\quad 86$

Mayer, Ph.; Revaz, C; Gloor, E.; De Grandi, P.: Aspects clinique et therapeutique

des sarcomes à faible degré de malignité de Гendomètre. A propos de 4 cas . . 89

Autorenregister 91 Schweizerische Gesellschaft für Gynäkologie und Geburtshilfe Bericht über die Jahresversammlung, 20. bis 22. Juni 1985 in Lugano

Société Suisse de Gynécologîe et Obstétrique Rapport de ГAssemblée annuelle, 20 au 22 juin 1985 à Lugano

Redaktor Rédacteur: E. Dreher, Bern

ISBN 3-8055-4242-9 